\title{
Deconjugation Characteristics of Ethylparaben Conjugates in Human Urine by Indirect Liquid Chromatography Tandem-mass Spectrometry
}

\author{
Yoshiyuki HARADA, ${ }^{1,2)}$, Shigeru SUZUKI ${ }^{1)}$ and Kazuko KODAMA ${ }^{1)}$ \\ ${ }^{1)}$ Graduate School of Bioscience and Biotechnology, Chubu University \\ (1200 Matsumoto, Kasugai, Aichi 487-8501 Japan) \\ ${ }^{2)}$ General Tokai Technology Center \\ (2-710 Inokoishi, Meito, Nagoya, Aichi 465-0021 Japan)
}

[Received July 25, 2017; Accepted January 9, 2018]

\begin{abstract}
Summary
An indirect quantitation method based on liquid chromatography tandem-mass spectrometry (LC/MS/MS) has been developed for the analysis of glucuronic acid and sulfuric acid conjugates of ethylparaben, for which standard reference reagents are not available. This method was then used for determining both the ethylparaben conjugates in human urine. The decay of these two conjugates in urine samples containing $\beta$-glucuronidase/arylsulfatase appeared to follow the first-order-like reaction kinetics; 4-hydroxybenzoic acid was not produced in the reaction. In addition, a multiple regression (MR) equation was obtained $\left(R_{a d j}^{2}=0.99, P<0.01\right)$ for the increase in ethylparaben concentration and decrease in the peak areas of its glucuronic acid and sulfuric acid conjugates. In the case of methylparaben and its glucuronic acid and sulfuric acid conjugates, a higher $P$-value was obtained in the MR equation for the glucuronic acid conjugate, owing to the low urinary concentration of methylparaben. Furthermore, this method was applied to determine the concentration of ethylparaben and its two conjugates in urine samples. Following the ingestion of an ethylparaben-containing supplement drink, $\sim 16 \%$ of ethylparaben was excreted as a mixture of ethylparaben and its glucuronic acid and sulfuric acid conjugates in the first morning urine of the subject on the following day.
\end{abstract}

Key words: Paraben, human urine, conjugate, indirect quantitation, liquid chromatography tandem-mass spectrometry

\section{INTRODUCTION}

Para-hydroxybenzoic acid esters (parabens) are widely used as antimicrobial agents and preservatives in cosmetics, pharmaceutical products, and foods; in Japan, approximately 4,000 tons of parabens were imported and produced in 2013 and $2014^{1}$. The concentrations of parabens in foods are controlled by the Food Sanitation $\mathrm{Act}^{2}$, which regulates the contents of ethyl-, iso-propyl-, $n$-propyl-, iso-butyl-, and $n$-butyl-parabens as food additives. For such regulations, the concentrations of these species are defined as equivalents of para-hydroxybenzoic acid, with examples including 0.25 and $0.10 \mathrm{~g} / \mathrm{L}$ in soy sauce and vinegar, and $0.1,0.012$, and $0.20 \mathrm{~g} / \mathrm{kg}$ in non-alcoholic beverages and syrup, rind of fruit or fruit vegetable, and fruit sauce..$^{2-4)}$. Parabens exhibit a broad spectrum of activity against molds, fungi, and bacteria, and show most potent activity against Gram-negative bacteria. Their antimicrobial properties increase in strength with increasing alkyl chain length. Parabens also exhibit estrogenic effects mediated by their phenolic hydroxyl groups, which bind in a similar manner to those of meso-hexoestrol ${ }^{5)}$. Indeed, Nishihara et al. ${ }^{6)}$ reported that parabens bind to estrogen receptors in vitro, while Oishi ${ }^{7}$ reported an in vivo decrease in sperm counts and testosterone levels in male rats exposed to parabens during fetal development.

However, paraben exposure routes and levels differ between humans and experimental animals. Upon exposure to parabens through dermal or oral uptake, parabens can be released from the human body into the environment, followed by metabolism within the organism' s cells, excretion into the urine, and subsequent disposal through the sewage system. To date, the levels of parabens and their metabolites have been measured in the environment ${ }^{8-11)}$, wastewater systems ${ }^{9}{ }^{16)}$, human cells, and urine samples ${ }^{12-15)}$. More specifically, the methyl- and propyl-paraben levels in environmental water were 262 and $25 \mathrm{ng} / \mathrm{L}$ in Aichi Japan ${ }^{9)}$ and 466 and $144 \mathrm{ng} / \mathrm{L}$ in Poland ${ }^{10)}$, respectively, while higher concentrations were detected in a river in Brazil, with methyl-, ethyl-, propyl-, and butyl-parabens being detected at levels of 8.0, 5.8, 13.1, and $15.1 \mu \mathrm{g} / \mathrm{L}$, respectively ${ }^{11)}$.

Urinary paraben levels have also been measured as intact species $^{12,13)}$, hydrolysis products ${ }^{14,16)}$, and conjugates of glucuronic and sulfuric acids ${ }^{14,15)}$. To identify such paraben conjugates, a method was previously reported where a precursor ion scan method was coupled with accurate mass analysis ${ }^{17}$; it was found that ethylparaben taken up into the human body was excreted in the urine as a mixture of the paraben itself, its hydrolyzed derivatives, and its conjugates of gluc- 
uronic and sulfuric acids, in addition to other unidentified species. Although it appeared that the majority of ethylparaben was conjugated with both glucuronic and sulfuric acid in urine, these conjugates could not be directly determined, as they are difficult to synthesize and are not readily commercially available, and therefore, no appropriate technique could be developed using such standards.

Thus, we herein present the development of an indirect quantitation method for the glucuronic acid and sulfuric acid conjugates of parabens based on liquid chromatography tandem-mass spectrometry (LC/MS/MS) and its subsequent application to the mass balance analysis of urinary metabolites of ethylparaben following the consumption of an ethylparaben-containing supplement drink.

\section{METHODS}

\section{Materials and reagents}

Mini-UniPrep filter vials were purchased from General Electric Company (Fairfield, CT, USA). ABS Elut-NEXUS cartridges (200 mg, $6 \mathrm{~mL}$ ) were obtained from Agilent Technologies (Santa Clara, CA, USA) and were washed sequentially with acetone, acetonitrile, and methanol (6 mL each), and then with distilled water $(12 \mathrm{~mL})$, prior to their use as clean-up cartridges. All 4-hydroxybenzoic acid methyl and ethyl esters (both from Biochemical Research) were purchased from Tokyo Chemical Industry (Tokyo, Japan). Methanol (for LC/MS) and acetone (for high-performance liquid chromatography, HPLC) were obtained from Kanto Chemical (Tokyo, Japan). Acetonitrile (for LC), distilled water (for LC/MS), $\beta$-glucuronidase/arylsulfatase (Cat. No. 10127060001, from Helix pomatia $\beta$-D-glucuronide glucuronosohydrolase/aryl-sulfate sulfohydrolase EC 3.2.1.31/EC 3.1.6.1), and methyl-tri- $O$-acetyl-1- $O$-(trichloroacetimidoyl)- $\alpha$-D-glucuronate (CAS92420-89-8, for Biochemical Research) were purchased from Merck-Millipore (Darmstadt, Germany), Wako Pure Chemical Industries (Osaka, Japan), Sigma-Aldrich (St Louis, MO, USA), and Junsei Chemical (Tokyo, Japan), respectively.

\section{Analysis}

The instrumental conditions employed for LC/MS/MS analysis are summarized in Table 1. A Waters Xevo TQ-S micro-triple quadrupole mass spectrometer coupled with a Waters ACQUITY ultraperformance liquid chromatography (UPLC) H-Class system (Milford MA, USA) was used for the identification and quantification of the target analytes. Chromatographic separation was performed on an ACQUITY UPLC HSS T3 column (I.D. $3.0 \mathrm{~mm} \times 100 \mathrm{~mm}, 1.8 \mu \mathrm{m}$, Waters). An aliquot of each extract ( $5 \mu \mathrm{L})$ was injected into the instrument, and negative ionization mode using selected reaction monitoring (SRM) was employed for analysis of the target analytes.

Multiple-regression analysis was used to determine the relationships of peak area decreases of paraben-glucuronic acid conjugate and paraben-sulfuric acid conjugate (explanatory variables) with the concentration increases of the corresponding parabens (objective variable). MS-Excel (2010) (Microsoft, Redmond, WA) was used for the calculation to minimize the standardized partial regression coefficient.

\section{Sample collection and preparation}

The first morning urine of one volunteer was collected following
Table 1 Instrumental conditions of liquid chromatographytandem mass spectrometry (LC/MS/MS)

\begin{tabular}{|c|c|c|c|}
\hline \multirow{2}{*}{ Instruments } & \multicolumn{3}{|c|}{ Waters ACQUITY UPLC H-class } \\
\hline & \multicolumn{3}{|c|}{ LC conditions } \\
\hline Column & \multicolumn{3}{|c|}{$\begin{array}{c}\text { Waters ACQUITY UPLC HSS T3 } 3.0 \times 100 \mathrm{~mm} \text {, } \\
1.8-\mu \mathrm{m}\end{array}$} \\
\hline Column Temp. & \multicolumn{3}{|c|}{$40^{\circ} \mathrm{C}$} \\
\hline \multirow[t]{3}{*}{ Mobile Phase } & \multicolumn{3}{|l|}{ A) Water } \\
\hline & \multicolumn{3}{|l|}{ B) $\mathrm{CH}_{3} \mathrm{CN}$} \\
\hline & \multicolumn{3}{|l|}{ C) $50 \mathrm{mM} \mathrm{CH}_{3} \mathrm{COONH}_{4}$} \\
\hline Gradient & Time $(\min ) \quad$ A (\%) & В $(\%)$ & $\mathrm{C}(\%)$ \\
\hline \multirow[t]{6}{*}{ profile } & 0 & 10 & 5 \\
\hline & 1.2 & 10 & 5 \\
\hline & 20 & 95 & 5 \\
\hline & 24 & 95 & 5 \\
\hline & 26 & 10 & 5 \\
\hline & 85 & 10 & 5 \\
\hline Flow rate & \multicolumn{3}{|c|}{$0.2 \mathrm{~mL} / \mathrm{min}$} \\
\hline Sample volume & \multicolumn{3}{|c|}{$5 \mu \mathrm{L}$} \\
\hline & \multicolumn{3}{|c|}{ Waters Xevo TQ-S micro } \\
\hline Ionization mode & \multicolumn{3}{|c|}{ ESI-negative } \\
\hline Acquisition mode & \multicolumn{3}{|c|}{ SRM } \\
\hline Capillary voltage & \multicolumn{3}{|c|}{$0.5 \mathrm{kV}$} \\
\hline Cone Voltage & \multicolumn{3}{|c|}{$50 \mathrm{~V}$} \\
\hline Desolvation Temp. & \multicolumn{3}{|c|}{$500^{\circ} \mathrm{C}$} \\
\hline Desolvation Flow & \multicolumn{3}{|c|}{$700 \mathrm{~L} / \mathrm{hr}$} \\
\hline Cone Flow & \multicolumn{3}{|c|}{$50 \mathrm{~L} / \mathrm{hr}$} \\
\hline Collision Energy MS & \multicolumn{3}{|c|}{$3 \mathrm{~V}$} \\
\hline
\end{tabular}

the consumption of an ethylparaben-containing supplement drink the previous night. This experiment was approved by the ethical committee of the Faculty of Bioscience and Biotechnology, Chubu University. The urine sample was divided into $10 \mathrm{~mL}$ aliquots and stored at $-20^{\circ} \mathrm{C}$ prior to sample preparation as outlined in Fig. 1. More specifically, the frozen samples were thawed at $\sim 20^{\circ} \mathrm{C}$, centrifuged at $3000 \mathrm{rpm}$ for $10 \mathrm{~min}$, and the supernatant solution was aspirated, homogenized, and divided into two aliquots of $50 \mathrm{~mL}$. The $\mathrm{pH}$ of each aliquot was adjusted using a small volume of $23 \mathrm{M}$ formic acid to either $\mathrm{pH} 4.3$ or 5.6 ; in both cases, high deconjugation reaction rates were individually obtained for the glucuronide and sulfate conjugates of ethylparaben in preliminary tests. Then, $\beta$-glucuronidase/arylsulfatase $(40 \mu \mathrm{L})$ was added to each resulting aliquot, which was divided into five portions of $10 \mathrm{~mL}$ in test tubes and placed in a water bath at $37^{\circ} \mathrm{C}$ to promote the gradual hydrolysis of the glucuronic acid and sulfuric acid conjugates of the parabens. An aliquot ( $1 \mathrm{~mL})$ of each solution was then sampled after $0,1,3,10,30,60,120$, and $180 \mathrm{~min}$, and acetonitrile $(1 \mathrm{~mL})$ was added to each aliquot prior to stirring for $30 \mathrm{~s}$ and subsequent clean-up using an ABS Elut-NEXUS cartridge. More specifically, the aliquot $(\sim 2 \mathrm{~mL})$ was passed through the cartridge, and the solution eluted from the cartridge using methanol $(8 \mathrm{~mL})$ was collected and diluted to $10 \mathrm{~mL}$ with methanol. Each sample was subjected to direct analysis of the parabens and indirect analysis of the paraben glucuronic acid and sulfuric acid conjugates by LC/MS/MS.

\section{RESULTS AND DISCUSSION}

\section{Ethylparaben recovery rate from urine}

The experimental recovery of methylparaben and ethylparaben from urine was carried out using seven $10 \mathrm{~mL}$ replicates of urine samples spiked with methylparaben and ethylparaben (10 ng each) in 


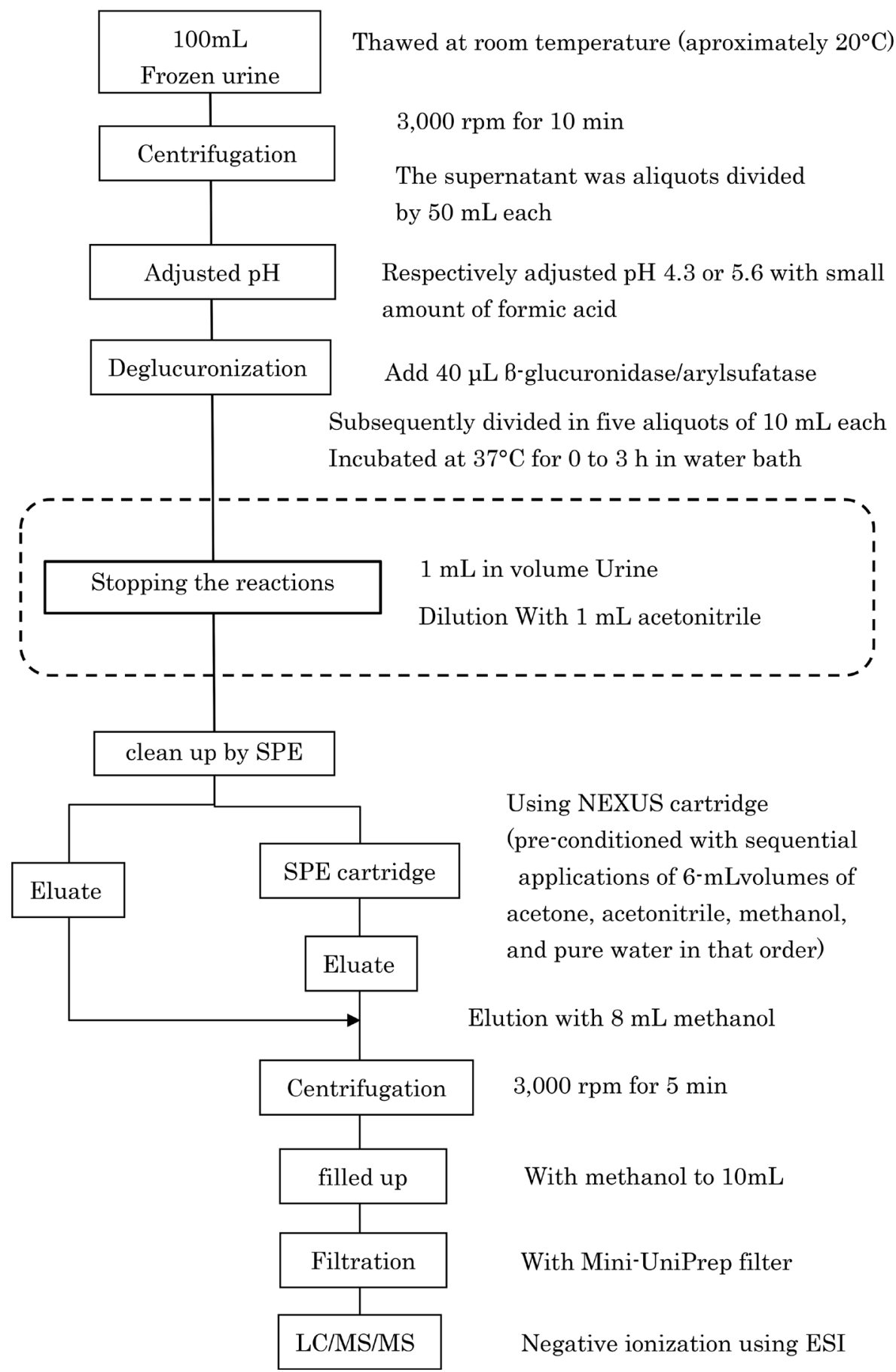

Fig. 1 Analysis scheme of parabens and their conjugates in human urine

addition to the urine control samples, which were prepared for analysis according to the scheme outlined in Fig. 1. The recovery rate was calculated by subtracting the concentrations of methylparaben and ethylparaben in the control sample from that of the spiked sample; the results are shown in Table 2. As indicated, the average recovery rate of methylparaben was $94 \%$ with a relative standard deviation (RSD) of 3.7\%, while that of ethylparaben was $114 \%$ with an RSD of $4.7 \%$. The limit of detection (LOD) and the limit of quantification (LOQ) of methylparaben were $13 \mu \mathrm{g} / \mathrm{L}$ and $38 \mu \mathrm{g} / \mathrm{L}$, respectively, and the LOD/LOQ of ethylparaben were, respectively, $22 \mu \mathrm{g} / \mathrm{L}$ and 66 $\mu \mathrm{g} / \mathrm{L}$. With these measures of accuracy and precision of the recovery rates, and the limits of detection (LOD), urinary concentration values of methylparaben and ethylparaben were used for indirect determination of their glucuronic acid and sulfuric acid conjugates.

Behavior of ethylparaben and its glucuronic acid and sulfuric acid conjugates in urine samples during the simultaneous deconjugation

The concentrations of ethylparaben and its glucuronic acid and sulfuric acid conjugates in the urine samples were measured 1, 3, 10, 30, 60, 120, and $180 \mathrm{~min}$ after the deconjugation reaction commenced. Representative SRM chromatograms illustrating the deconjugation of the two ethylparaben conjugates to produce ethylparaben in the urine samples after reaction times of 0 and $180 \mathrm{~min}$ are shown in Fig. 2. As 
Table 2 Recovery of methylparaben (M.P.) and ethylparaben (E.P.) from urine sample ${ }^{* 1}(n=7)$

\begin{tabular}{lcc}
\hline Substance name & $\begin{array}{c}\text { Methyl } \\
\text { paraben }\end{array}$ & $\begin{array}{c}\text { Ethyl } \\
\text { paraben }\end{array}$ \\
\hline Control urine Avg. $(\mu \mathrm{g} / \mathrm{L})$ & 7.9 & 25 \\
\hline \hline Spiked amount [ng] & 100 & 100 \\
Recovered Avg. $(\mu \mathrm{g} / \mathrm{L})$ & 102 & 140 \\
Recovery Avg. [\%] & 94 & 115 \\
$\operatorname{RSD}(\%)$ & 3.7 & 4.7 \\
$\mathrm{LOD}(\mu \mathrm{g} / \mathrm{L}) * 2$ & 13 & 22 \\
$\mathrm{LOQ}(\mu \mathrm{g} / \mathrm{L}) * 3$ & 38 & 66 \\
\hline
\end{tabular}

*1 The recovery experiment was performed by adding methylparaben (M.P.) and ethylparaben (E.P.) to control urine.

$* 2$ LOD $=3.29 \times$ S.D. (S.D.: standard deviation obtained with the present study)

${ }^{*} \mathrm{LOQ}=3.04 \times \mathrm{LOD}$ indicated, the production of 4-hydroxybenzoic acid was not observed during the deconjugation reaction. In addition, Fig. 3 shows plots of the peak areas corresponding to the two ethylparaben conjugates and the ethylparaben concentrations against time for the deconjugation reaction at $\mathrm{pH} 4.3$ and $\mathrm{pH}$ 5.6. Indeed, clear exponential decays were observed between reaction times of 10-180 min. The lower decay rates of the conjugates within the initial $10 \mathrm{~min}$ of the reaction were likely due to the delay in reaching the designated reaction temperature. However, the decay rate did not influence the relationship between the increase in ethylparaben concentration and the decrease in peak areas of the glucuronic acid and sulfuric acid conjugates, and the lower decay rates were helpful in accurately measuring the changes in concentration and peak areas over the initial $10 \mathrm{~min}$, in which five replicates of the urine samples were measured after 0,3 , and 10 min. Interestingly, the decay rates of the sulfuric acid conjugate were

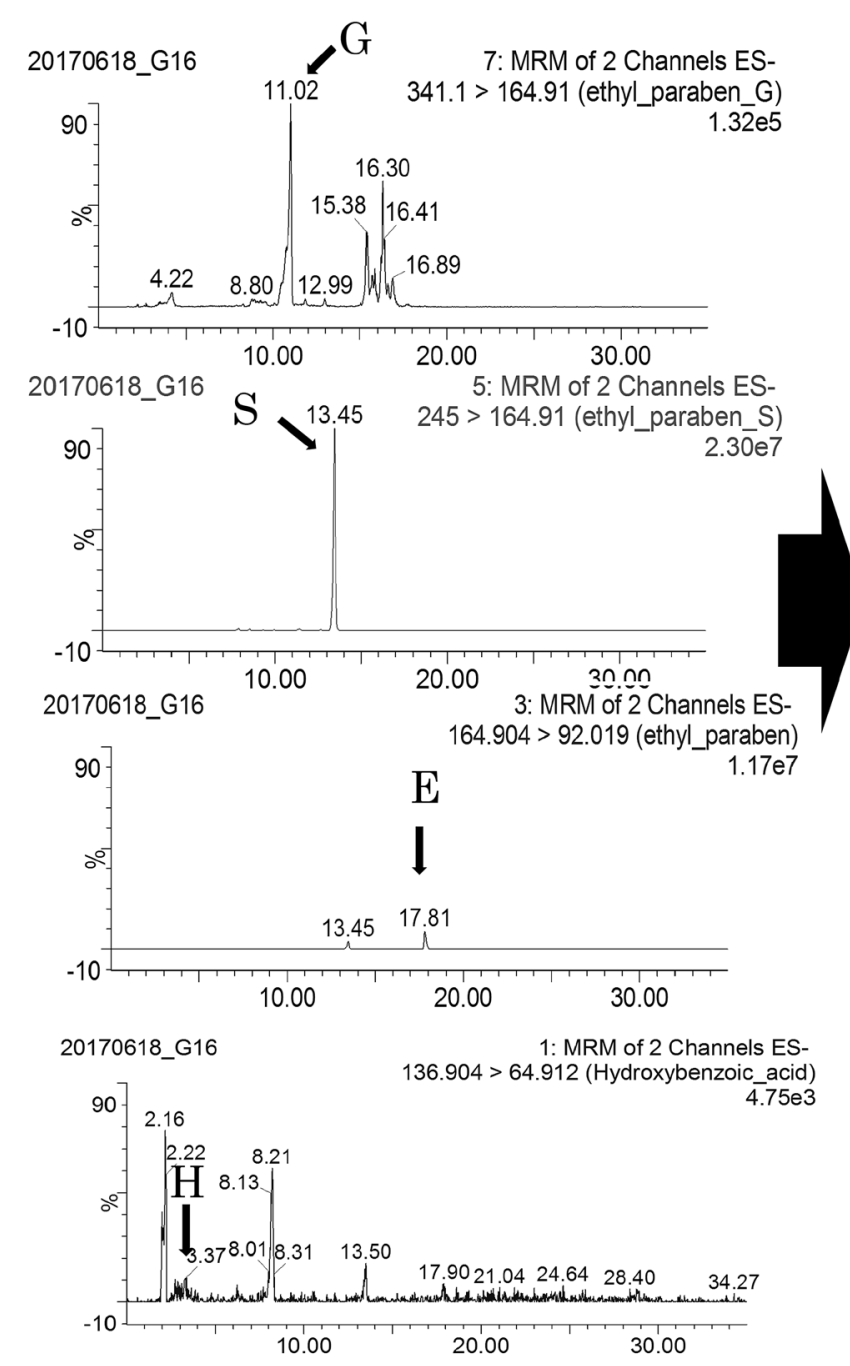

Before the deconjugation (0 min)
20170618_G56 7: MRM of 2 Channels ES-

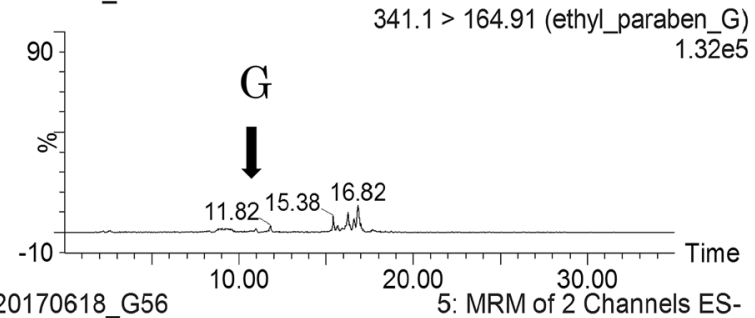

$245>164.91$ (ethyl_paraben_S)

$2.3 \overline{0} \mathrm{e} 7$

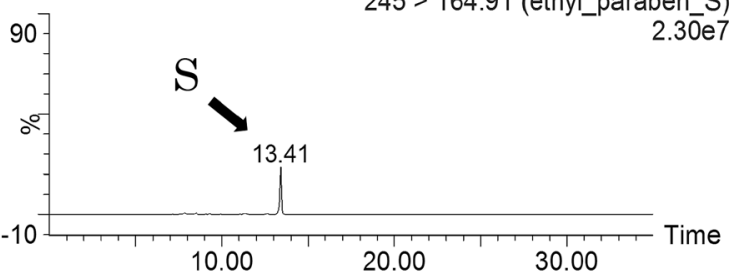

20170618_G56 3: MRM of 2 Channels ES-
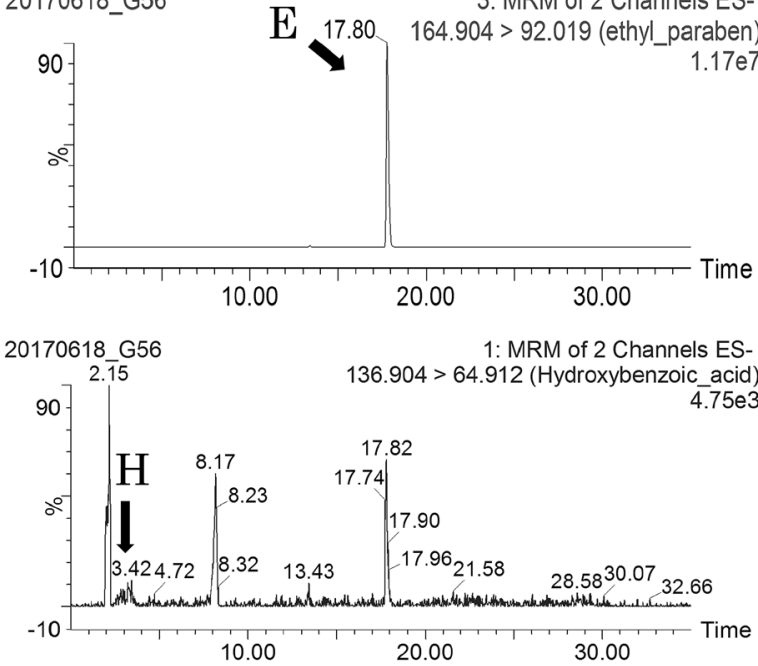

After the deconjugation (180min)

\section{E : Ethylparaben $\quad$ G: Glucuronic acid conjugate of ethylparaben \\ $\mathrm{H}:$ 4-hydroxybenzoic acid $\mathrm{S}:$ Sulfuric acid conjugate of ethylparaben}

Fig. 2 SRM chromatograms illustrating before and after the deconjugation of the two ethylparaben conjugates to produce the intact ethylparaben in urine samples

The deconjugation reaction was performed at $\mathrm{pH} 5.6$ 
higher at $\mathrm{pH} 5.6$ than at $\mathrm{pH} 4.3$, while those of the glucuronic acid conjugate were comparable under both $\mathrm{pH}$ conditions. In addition, the exponential decays observed for the two ethylparaben conjugates suggest that both conjugates decayed according to first-order-like reactions, and that the rate of glucuronic acid conjugate decay was higher than that of the sulfuric acid conjugate. A linear increase in the logarithmic concentration, i.e., an exponential increase in the concentration, of ethylparaben was also observed, as expected from the exponential decay of the ethylparaben conjugates.

The observed results therefore suggest that the glucuronic acid and sulfuric acid conjugates of ethylparaben in the urine samples were successfully deconjugated in the presence of $\beta$-glucuronidase/ arylsulfatase to produce ethylparaben via two simultaneous firstorder-like reactions, with no 4-hydroxybenzoic acid production being observed in either case. Although the deconjugation reaction was performed at $\mathrm{pH} 4.3$ and $\mathrm{pH} 5.6$, the deconjugation reaction rates of the sulfuric acid conjugates were very low at $\mathrm{pH} 4.3$, while at $\mathrm{pH} 5.6$, the deconjugation reaction rates were good enough for both the ethylparaben conjugates. Therefore, all further deconjugation experiments using indirect LC/MS/MS were performed at $\mathrm{pH}$ 5.6.

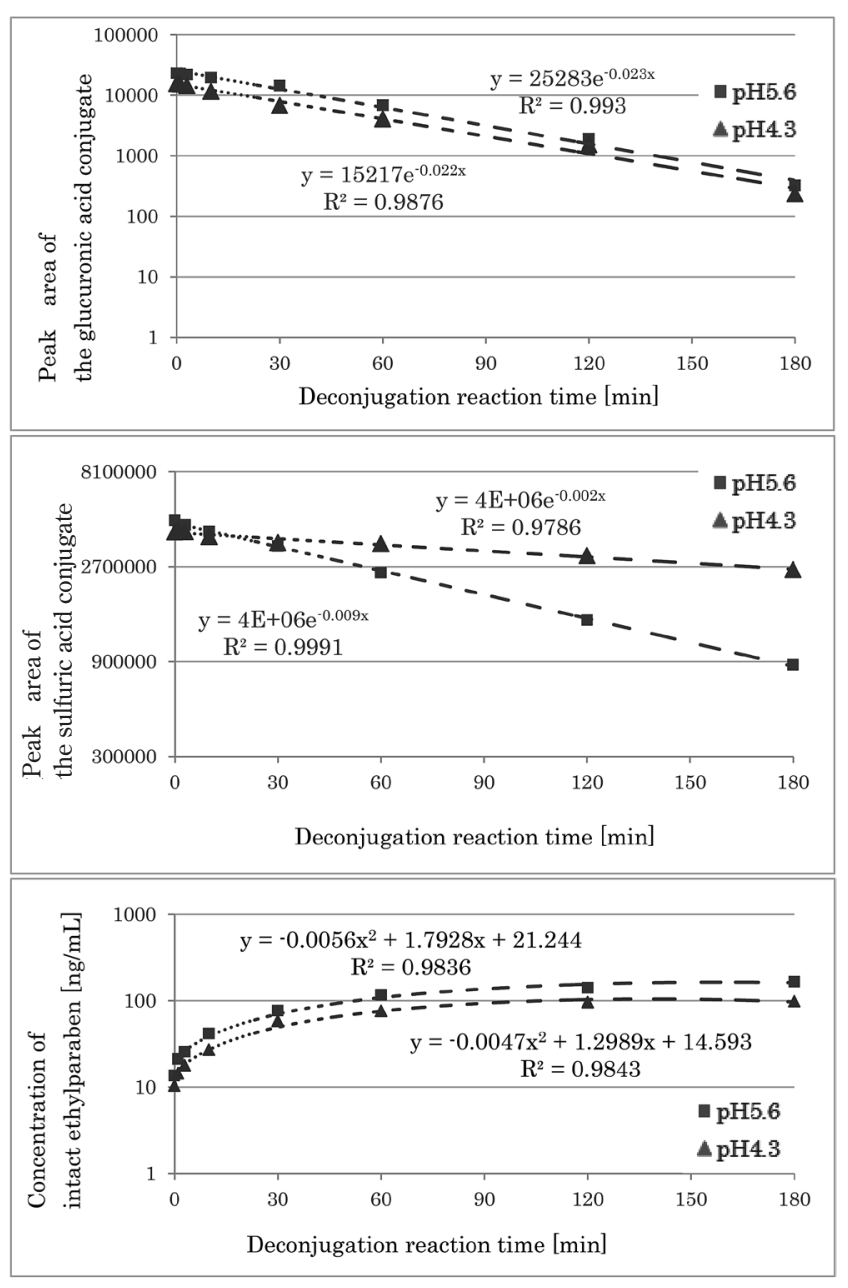

Fig. 3 Peak areas corresponding to the two ethylparaben conjugates and concentrations of intact ethylparaben plotted against the simultaneous deconjugation reaction time

The deconjugation reaction was performed at $\mathrm{pH} 5.6$

\section{Indirect LC/MS/MS method for determination of the glucuronic acid and sulfuric acid conjugates of ethylparaben}

Based on the assumption that the decay of the glucuronic acid and sulfuric acid conjugates of ethylparaben took place through simultaneous first-order reactions, multiple regression analysis (MRA) was performed (see Table 3) to estimate the increase in ethylparaben concentration using decreases in the conjugate peak areas as explanatory variables. The multiple regression equation (1) shown in Table 3 was obtained $\left(R_{a d j}^{2}=0.99, P<0.01\right)$ for the increase in ethylparaben concentration $\left(\mathrm{C}_{\mathrm{ep}}\right)$ and the decrease in its conjugate peak areas (i.e., glucuronic acid $\left(\mathrm{A}_{\mathrm{e}-\mathrm{g}}\right)$ and sulfuric acid $\left(\mathrm{A}_{\mathrm{e}-\mathrm{s}}\right)$ ). However, in general, the coefficients of $A_{e-g}, A_{e-s}$, and the constant of 5.9 in equation (1) are dependent on analytical conditions and urine samples. Thus, equation (1) is generally written as follows:

$\mathrm{C}_{\mathrm{ep}}=\mathrm{A} * \mathrm{~A}_{\mathrm{e}-\mathrm{g}}+\mathrm{B} * \mathrm{~A}_{\mathrm{e}-\mathrm{s}}+\mathrm{C} \quad(\mathrm{A}, \mathrm{B}$, and $\mathrm{C}$ are constant $) \quad(1)^{\prime}$

Coefficients $\mathrm{A}$ and $\mathrm{B}$, and constant $\mathrm{C}$ are generally determined with a minimum of three values of $\mathrm{C}_{\mathrm{ep}}, \mathrm{A}_{\mathrm{e}-\mathrm{g}}$, and $\mathrm{A}_{\mathrm{e}-\mathrm{s}}$ from SRM measurements. Then, using equation $(1)^{\prime}$, for which the coefficients and constant $\mathrm{A}, \mathrm{B}$, and $\mathrm{C}$ have been determined, the concentrations of the glucuronic acid and sulfuric acid conjugates of ethyl paraben can be indirectly determined.

Similarly, the urinary concentrations of the glucuronic acid and sulfuric acid conjugates of methylparaben can also be indirectly estimated by solving simultaneous equations between the decrease in peak areas of the two conjugates and the increase in the quantified concentration of methylparaben (see Table 3 ). In this case, the multiple regression equation (2) (shown in Table 3) was obtained for the relationship between the methylparaben concentration $\left(C_{m p}\right)$ and the peak areas of the glucuronic acid conjugate $\left(\mathrm{A}_{\mathrm{m}-\mathrm{g}}\right)$ and the sulfuric acid conjugate $\left(\mathrm{A}_{\mathrm{m}-\mathrm{s}}\right)\left(R_{a d j}^{2}=0.96, P<0.01\right.$ for sulfuric acid, $P=0.29$ for glucuronic acid). The higher $P$-value for the glucuronic acid conjugate was caused by the small peak areas observed, because methylparaben was not intentionally ingested in the experiment, i.e., the higher $P$-value for the glucuronic acid conjugate of methyl paraben was due to the low concentration and the low sensitivity.

Fig. 4 shows a scatter plot of the ethylparaben equivalent concentration decreases calculated from the peak area decreases of the glucuronic and sulfuric conjugates of ethylparaben with equation (1) and the increases in ethylparaben concentrations. Similarly, a scatter plot of the methylparaben equivalent concentration decreases calculated with equation (2) and the increases in methylparaben concentrations are shown in Fig. 5. Excellent correlations were obtained between the paraben equivalent concentration decreases and paraben concentration increases for both ethylparaben $\left(R_{a d j}^{2}=0.99\right.$, abundance ratio $=$ $0.99)$ and methylparaben $\left(R_{a d j}^{2}=0.96\right.$, abundance ratio $\left.=0.96\right)$. Therefore, it could be concluded that the concentrations of the methyl- and ethylparaben conjugates can be indirectly quantitated by the method presented herein.

Finally, the concentrations of the glucuronic and sulfuric acid conjugates of ethylparaben present in the first morning urine of one volunteer were measured using the indirect quantitation method following the ingestion of an ethylparaben-containing supplement drink. All measurements were repeated five times and an average concentration equivalent to ethylparaben was taken to obtain ethylparaben, glucuronic acid conjugate, and sulfuric conjugate of $140 \mathrm{ng} / \mathrm{mL}$ (RSD 3.3\%), $950 \mathrm{ng} / \mathrm{mL}$ (RSD 3.4\%), and $620 \mathrm{ng} / \mathrm{mL}$ (RSD 1.2\%), respective- 
Table 3 Results of MRA between concentration increases of methyl and ethyl parabens and peak area decreases of those conjugates of glucuronic acid and sulfuric acid $(\mathrm{N}=35)$

\begin{tabular}{|c|c|c|c|c|c|c|}
\hline $\begin{array}{l}\text { multiple } \\
\text { regression }\end{array}$ & \multicolumn{5}{|c|}{$\begin{array}{l}C_{e p}{ }^{* 1}=0.0041 A_{e-g}{ }^{* 2}+0.000013 A_{e-s}{ }^{* 3}+5.9 \\
C_{m p}{ }^{* 4}=0.0019 A_{m-g}{ }^{* 5}+0.000028 A_{m-s}{ }^{* 6}+0.019\end{array}$} & $\begin{array}{l}(1) \\
(2)\end{array}$ \\
\hline $\begin{array}{l}\text { objective } \\
\text { variable }\end{array}$ & $\begin{array}{c}\text { explanatory } \\
\text { variables }\end{array}$ & $\begin{array}{c}\text { partial } \\
\text { regression } \\
\text { coefficient }\end{array}$ & $\begin{array}{l}\text { standardized } \\
\text { partial } \\
\text { regression } \\
\text { coefficient }\end{array}$ & T- value & $\mathrm{p}$ - value & $\begin{array}{c}\mathrm{R}^{2} \\
\text { adjusted }\end{array}$ \\
\hline \multirow{3}{*}{$\mathrm{C}_{\mathrm{ep}}$} & $A_{e-g}$ & 0.0041 & 0.67 & 6.9 & 0.000000089 & \\
\hline & $A_{e-s}$ & 0.000013 & 0.33 & 3.4 & 0.0021 & 0.9869 \\
\hline & constants & 5.9 & - & 3.5 & 0.0013 & \\
\hline \multirow{3}{*}{$\mathrm{C}_{\mathrm{mp}}$} & $A_{m-g}$ & 0.0019 & 0.25 & 1.1 & 0.29 & \\
\hline & $A_{m-s}$ & 0.000028 & 0.73 & 3.2 & 0.0034 & 0.9553 \\
\hline & constants & 0.019 & - & 0.097 & 0.92 & \\
\hline
\end{tabular}

The deconjugation reaction was performed under $\mathrm{pH} 5.6$.

* 1 : increase in ethylparaben concentration.

*2 and *3: decrease in the ethylparaben conjugate peak areas of glucuronic acid and sulfuric acid, respectively.

* 4 : increase in methylparaben concentration.

*5 and *6: decrease in the methylparaben conjugate peak areas of glucuronic acid and sulfuric acid, respectively.

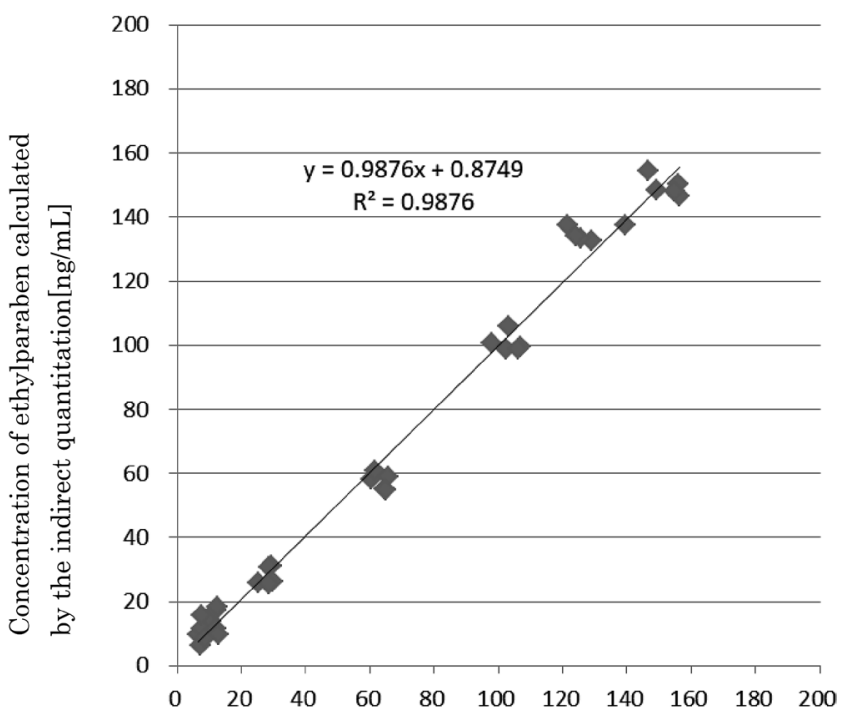

Concentration of ethylparaben by measurement $[\mathrm{ng} / \mathrm{mL}]$

Fig. 4 Scatter plots of calculated and measured values of the ethylparaben concentration increases by deconjugations of its glucuronic acid and sulfuric acid conjugates

The deconjugation reaction was performed at $\mathrm{pH} 5.6$

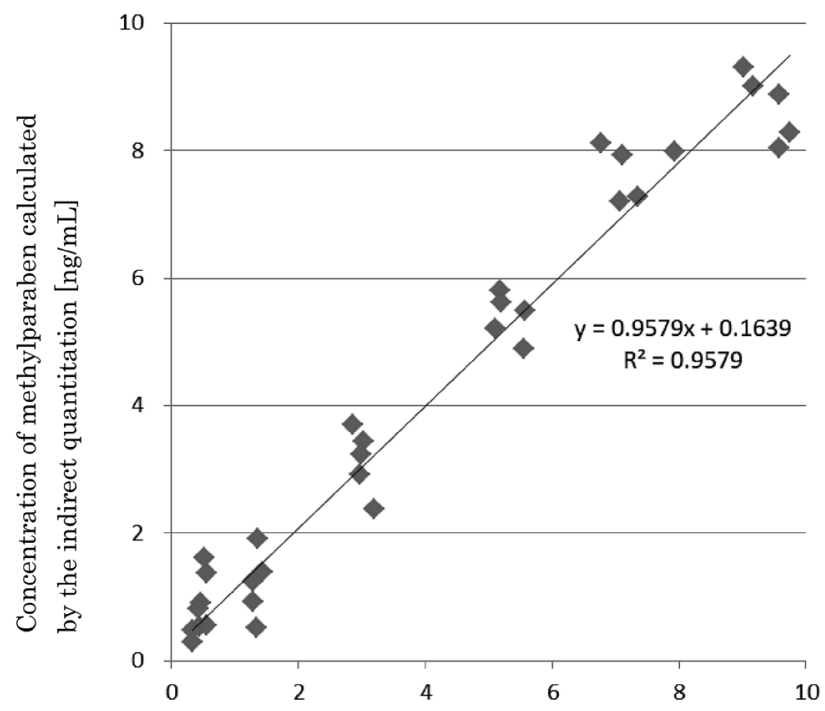

Concentration of methylparaben by measurement $[\mathrm{ng} / \mathrm{mL}]$

Fig. 5 Scatter plots of calculated and measured values of the methylparaben concentration increases by deconjugations of its glucuronic acid and sulfuric acid conjugates

The deconjugation reaction was performed at $\mathrm{pH} 5.6$ 
ly. The total quantity of ethylparaben and its two conjugates excreted in the first morning urine equivalent concentration to ethylparaben was thus $780 \mu \mathrm{g}$ (RSD 2.3\%), which constituted $16 \%$ of the ethylparaben ingested from the supplement drink the previous night. However, detailed data relating to the behavior of the ingested parabens were limited, owing to the low urinary concentrations of methylparaben and other parabens, and so it is apparent that additional experiments using more sensitive measurement techniques will be required to interpret the conjugation process and the behavior of the conjugates in greater detail.

\section{CONCLUSION}

We herein reported the development of an indirect quantitation method based on LC/MS/MS for determining the concentration of the glucuronic acid and sulfuric acid conjugates of ethylparaben, for which standard reference reagents are not available. The two ethylparaben conjugates were found to undergo deconjugation using $\beta$-glucuronidase/arylsulfatase to produce ethylparaben by two simultaneous first-order-like reactions. The production of 4-hydroxybenzoic acid was not observed in either case. In addition, indirect quantitation of the two conjugates was successfully performed by analyzing the relationship between the increase in ethylparaben concentration and the decrease in conjugate peak areas over a range of reaction times $\left(R_{a d j}^{2}=0.987, \mathrm{P}<0.01\right)$. Finally, this method was tested using a mixed reagent of $\beta$-glucuronidase / allylsulfatase, assuming a small amount of urine sample. This method, which enables calculation of concentrations of two kinds of conjugates by one line of analysis operation, is a method in which the amount of ethylparaben and its glucuronic acid and sulfuric acid conjugate thereof in the urine sample after ingestion of the ethylparaben-containing beverage by human subjects was successfully applied to determine. These results are of particular importance as our developed method can be used in the analysis of parabens and their conjugates in biological samples, to ultimately achieve improved control of the permitted paraben levels in foodstuffs, pharmaceutical products, and cosmetics. The method may contribute to a study such as the Japan Environment and Children's Study, in which exposure measurements of chemicals have been performed with limited sample sizes. However, additional studies are required to elucidate the specific metabolic behavior of such compounds. These studies will be carried out in the near future, and the results will be reported in due course.

\section{ACKNOWLEDGEMENTS}

The author would like to thank Mr. Tsuchiya for his support with the research activities.

\section{REFERENCES}

1) Publication of the name and notified volume of substances whose total manufacturing and import volume, (FY2013), and (FY2014), hydroxybenzoic acid alkyl ester $(\mathrm{C}=1-22)$, Monitoring Chemical Substances by the Act on the Evaluation of Chemical Substances and Regulation of Their Manufacture, etc., Ministry of Economy, Trade and Industry (in Japanese) (2015)

2) Food Sanitation Act Article 11 (1), Ministry of Health, Labour and Welfare, Act No. 233 (1947)

3) Smolinske, S.C.: "Handbook of Food, Drug, and Cosmetic Excipients.” CRC Press, Boca Raton, FL, USA, pp 251-258 (1992)

4) Specifications and Standards for Foods, Food Additives, etc. Under the Food Sanitation Act (Abstract) 2010, JETRO (2011)

5) Byford, J.R., Shaw, L.E., Drew, M.G., Pope, G.S., Sauer, M.J. and Darbre, P.D.: Oestrogenic activity of parabens in MCF7 human breast cancer cells. J. Steroid Biochem. Mol. Biol., 80, 49-60 (2002)

6) Nishihara, T., Nishikawa, J., Kanayama, T., Dakeyama, F., Saito, K., Imagawa, M., Takatori, S., Kitagawa, Y., Hori, S. and Utsumi, U.: Estrogenic activities of 517 chemicals by yeast two-hybrid assay. J. Health Sci., 46, 282-298 (2000)

7) Oishi, S.: Effects of propyl paraben on the male reproductive system. Food Chem. Toxicol., 40, 1807-1813 (2002)

8) Kimura, K., Kameda, Y., Watanabe, S. and Masunaga, S.: Detection and seasonal variation of preservatives in rivers in Saitama City, Proceedings of 46th Annual Conference of Japan Society on Water Environment, p 525, Tokyo (2012)

9) Harada, Y. and Suzuki, S.: A study on parabens in water environment by LC/MS/MS, 45th Annual Meeting of Union of Chemistry-Related Societies in Chubu Area, Kasugai (2014)

10) Zgoła-Grześkowiak, A., Jeszka-Skowron, M., CzarczyńskaGoślińska, B. and Grześkowiak, T.: Determination of parabens in Polish river and lake water as a function of season. Anal. Lett., 49, 1734-1747 (2016)

11) Galinaro, C.A., Pereira, F.M. and Vieira, E.M.: Determination of parabens in surface water from Mogi Guaçu River (São Paulo, Brazil) using dispersive liquid-liquid microextraction based on low density solvent and LC-DAD. J. Braz. Chem. Soc., 26, 22052213 (2015)

12) Ma, W.L., Wang, L., Guo, Y., Liu, L.Y., Qi, H., Zhu, N.Z., Gao, C.J., Li, Y.F. and Kannan, K.: Urinary concentrations of parabens in Chinese young adults: Implications for human exposure. Arch. Environ. Contam. Toxicol., 65, 611-618 (2013)

13) Cristina Jardim, V., de Paula Melo, L., Soares Domingues, D. and Costa Queiroz, M.E.: Determination of parabens in urine samples by microextraction using packed sorbent and ultra-performance liquid chromatography coupled to tandem mass spectrometry. $J$. Chromatogr. B Analyt. Technol. Biomed. Life Sci., 1, 35-41 (2015)

14) Abbas, S., Greige-Gerges, H., Karam, N., Piet, M.H., Netter, P., Magdalou, J. and Jacques, M.: Metabolism of parabens (4-hydroxybenzoic acid esters) by hepatic esterases and UDP-glucuronosyltransferases in man, Drug Metab. Pharmacokinet., 2, 568577 (2010)

15) Shirai, S., Suzuki, Y., Yoshinaga, J., Shiraishi, H. and Mizumoto, Y.: Urinary excretion of parabens in pregnant Japanese women, Reprod. Toxicol., 35, 96-101 (2013)

16) Wang, W. and Kannan, K.: Fate of parabens and their metabolites in two wastewater treatment plants in New York State, United States. Environ. Sci. Technol., 50, 1174-1181 (2016)

17) Kodama, K., Suzuki, S. and Harada, Y.: Separation and identification of paraben conjugates in human urine by liquid chromatography/tandem mass spectrometry combining precursor ion scan and accurate mass analysis. J. Environ. Chem., 27, 1-8 (2017) 